\title{
MicroRNA-34a Inhibition of the TLR Signaling Pathway Via CXCL10 Suppresses Breast Cancer Cell Invasion and Migration
}

\section{Min $\mathrm{Xu}^{\mathrm{a}} \quad$ Dong Li ${ }^{\mathrm{b}} \quad$ Chen Yang ${ }^{\mathrm{c}} \quad$ Jian-Song Ji}

aKey Laboratory of Imaging Diagnosis and Minimally Invasive Intervention Research, The Fifth Affiliated Hospital of Wenzhou Medical University, Affiliated Lishui Hospital of Zhejiang University, Lishui Municipal Central Hospital, Lishui, ${ }^{b}$ Clinical Laboratory, Shanghai Tongji Hospital, Tongji University School of Medicine, Shanghai 'Clinical Laboratory, Suzhou Municipal Hospital, the Affiliated Suzhou Hospital of Nanjing Medical University, China 